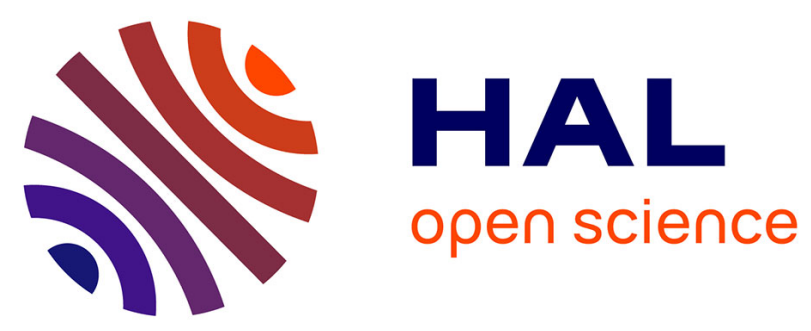

\title{
Evaluation of the impact of a scanner prototype on proton CT and helium CT image quality and dose efficiency with Monte Carlo simulation
}

Stefanie Götz, Jannis Dickmann, Simon Rit, Nils Krah, Feriel Khellaf, Reinhard W Schulte, Katia Parodi, George Dedes, Guillaume Landry

\section{To cite this version:}

Stefanie Götz, Jannis Dickmann, Simon Rit, Nils Krah, Feriel Khellaf, et al.. Evaluation of the impact of a scanner prototype on proton CT and helium CT image quality and dose efficiency with Monte Carlo simulation. Physics in Medicine and Biology, 2022, 67, 10.1088/1361-6560/ac4fa4 . hal-03583696

\section{HAL Id: hal-03583696 https://hal.science/hal-03583696}

Submitted on 22 Feb 2022

HAL is a multi-disciplinary open access archive for the deposit and dissemination of scientific research documents, whether they are published or not. The documents may come from teaching and research institutions in France or abroad, or from public or private research centers.
L'archive ouverte pluridisciplinaire HAL, est destinée au dépôt et à la diffusion de documents scientifiques de niveau recherche, publiés ou non, émanant des établissements d'enseignement et de recherche français ou étrangers, des laboratoires publics ou privés. 


\title{
PAPER
}

CrossMark

\section{Evaluation of the impact of a scanner prototype on proton CT and}

OPEN ACCESS

RECEIVED

8 November 2021

REVISED

18 January 2022

ACCEPTED FOR PUBLICATION

27 January 2022

PUBLISHED

21 February 2022

Original content from this work may be used under the terms of the Creative

Commons Attribution 4.0 licence.

Any further distribution of this work must maintain

attribution to the author(s) and the title of

the work, journal citation and DOI. helium CT image quality and dose efficiency with Monte Carlo simulation

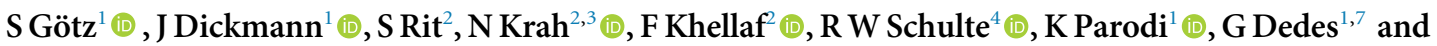
G Landry ${ }^{1,5,6,7}$ (1)

1 Department of Medical Physics, Fakultät für Physik, Ludwig-Maximilians-Universität München (LMU Munich), D-85748 Garching bei München, Germany

2 University of Lyon, INSA-Lyon, Unversité Claude Bernard Lyon 1, UJM-Saint Etienne, CNRS, Inserm, CREATIS, UMR 5220, U1294 F-69373, Lyon, France

IP2I, UMR 5822 F-69622, Villeurbanne, France

4 Division of Biomedical Engineering Sciences, Loma Linda University, Loma Linda, CA 92354, United States of America

5 Department of Radiation Oncology, University Hospital, LMU Munich, D-81377 Munich, Germany

6 German Cancer Consortium (DKTK), D-81377 Munich, Germany

Senior authorship is shared equally.

E-mail: guillaume.landry@med.uni-muenchen.de

Keywords: proton CT, relative stopping power, helium CT, image quality, Monte Carlo, spatial resolution

\begin{abstract}
Objective. The use of ion computed tomography (CT) promises to yield improved relative stopping power (RSP) estimation as input to particle therapy treatment planning. Recently, proton CT (pCT) has been shown to yield RSP accuracy on par with state-of-the-art x-ray dual energy CT. There are however concerns that the lower spatial resolution of pCT compared to $\mathrm{x}$-ray CT may limit its potential, which has spurred interest in the use of helium ion CT (HeCT). The goal of this study was to investigate image quality of $\mathrm{pCT}$ and $\mathrm{HeCT}$ in terms of noise, spatial resolution, $\mathrm{RSP}$ accuracy and imaging dose using a detailed Monte Carlo (MC) model of an existing ion CT prototype. Approach. Three phantoms were used in simulated pCT and HeCT scans allowing estimation of noise, spatial resolution and the scoring of dose. An additional phantom was used to evaluate RSP accuracy. The imaging dose required to achieve the same image noise in a water and a head phantom was estimated at both native spatial resolution, and in a scenario where the HeCT spatial resolution was reduced and matched to that of $\mathrm{pCT}$ using Hann windowing of the reconstruction filter. A variance reconstruction formalism was adapted to account for Hann windowing. Main results. We confirmed that the scanner prototype would produce higher spatial resolution for HeCT than pCT by a factor $1.8\left(0.86 \mathrm{lp} \mathrm{mm}^{-1}\right.$ versus $0.48 \mathrm{lp} \mathrm{mm}^{-1}$ at the center of a $20 \mathrm{~cm}$ water phantom). At native resolution, $\mathrm{HeCT}$ required a factor 2.9 more dose than pCT to achieve the same noise, while at matched resolution, HeCT required only $38 \%$ of the pCT dose. Finally, RSP mean absolute percent error (MAPE) was found to be $0.59 \%$ for $\mathrm{pCT}$ and $0.67 \%$ for HeCT. Significance. This work compared the imaging performance of $\mathrm{pCT}$ and $\mathrm{HeCT}$ when using an existing scanner prototype, with the spatial resolution advantage of $\mathrm{HeCT}$ coming at the cost of increased dose. When matching spatial resolution via Hann windowing, HeCT had a substantial dose advantage. Both modalities provided state-of-the-art RSP MAPE. HeCT might therefore help reduce the dose exposure of patients with comparable image noise to pCT, enhanced spatial resolution and acceptable RSP accuracy at the same time.
\end{abstract}

\section{Introduction}

The necessity for highly accurate stopping power relative to water (RSP) voxel maps in modern particle therapy treatment planning spurred the investigation and development of new imaging modalities. In current clinical 
practice, those RSP maps are commonly determined from single energy x-ray computed tomography (CT) scans, which yield uncertainties of up to 3.5\% (Paganetti 2012, Yang et al 2012). This is caused by the conversion of the photon attenuation coefficient to the corresponding RSP of particles using look-up tables and stoichiometric calibrations. Developments in dual-energy x-ray CT (Yang et al 2010) allowed reducing errors to around 1\% (Hünemohr et al 2013, Hudobivnik et al 2016, Taasti et al 2017, Wohlfahrt et al 2017, Bär et al 2018, Berthold et al 2021, Niepel et al 2021).

Another imaging technique which provides a more direct access to RSP is ion CT. With this imaging modality, a water-equivalent path length (WEPL), which is the line integral of the RSP, is determined from the particles' residual energy. Since the idea was originally proposed by Cormack (1964) and first realised by Hanson (1979), several prototypes were recently developed and are now under investigation for proton and heavier ion CT (Coutrakon et al 2013, Rinaldi et al 2013, Johnson et al 2016, Sadrozinski et al 2016, Meyer et al 2017, Pettersen et al 2017, Esposito et al 2018, Civinini et al 2020). One of those prototypes is the phase-II proton CT (pCT) scanner (Bashkirov et al 2016, Johnson et al 2016) located at the Northwestern Medicine Chicago proton center. This scanner, which provides comparable RSP accuracy to clinical dual-energy x-ray CT technology (Dedes et al 2019), was additionally modelled in a Geant4 Monte Carlo simulation platform (Giacometti et al 2017b), which is a well-established approach for the development, characterisation and optimisation of new radiotherapy devices (Park et al 2021). This Geant 4 implementation has been validated against experimental data (Giacometti et al 2017b, Dedes et al 2019, Dickmann et al 2019) and allows to gain insight into the impact of the scanner design on image quality (Dedes et al 2019,2020). The combination of scanner and simulation was already successfully used for the development of new approaches such as the application of fluence modulation to pCT (Dedes 2017, Dickmann et al 2020a, 2020b, 2021b) as well as several correction methods (Dickmann et al 2021a, 2021c). Moreover, the variance reconstruction of Rädler et al (2018) was validated for protons for this scanner (Dickmann et al 2019). The phase-II pCT scanner was further successfully used with helium ions and first experiments to investigate image artefacts, spatial resolution, RSP as well as range prediction accuracy have been performed (Volz et al 2018, Bär et al 2021, Volz et al 2021).

However, further research on helium CT (HeCT) has so far mainly focused on studying the physical properties of the particles and their impact on imaging under ideal (Hansen et al 2014, Piersimoni et al 2018, Meyer et al 2019,2021) and simplified conditions (Collins-Fekete et al 2021). Those results might be different within a practical implementation due to effects introduced by a realistic scanner setup.

This work provides an investigation of $\mathrm{HeCT}$ under the consideration of realistic conditions as well as a comparison to pCT. For this purpose, we simulated the entire imaging procedure for a set of different phantoms using the Geant4 implementation of the phase-II pCT scanner (Bashkirov et al 2016, Johnson et al 2016, Giacometti et al 2017b, Dickmann et al 2019). The distance driven binning (DDB) filtered backprojection (FBP) (Rit et al 2013) reconstructions of RSP and variance maps of the phantoms were the basis for our subsequent evaluation of $\mathrm{PCT}$ and $\mathrm{HeCT}$ in terms of dose, image variance and spatial resolution. The implementation of Hann windowing in the reconstruction filter made modification and matching of these quality parameters possible via tuning of the cutoff frequency. We thus additionally present a procedure which allows a fair comparison between protons and helium ions in terms of a single final criterion, similarly to Tilley et al (2015) for x-ray CT and Collins-Fekete et al (2021) for ion CT. Moreover, the accuracy of the RSP of both imaging modalities, pCT and HeCT, was evaluated for a set of various phantom materials.

\section{Materials and methods}

\subsection{Simulation framework}

Data for this study was generated using the Geant4 (version 10.05.p01) Monte Carlo simulation platform (Agostinelli et al 2003) of the phase-II pCT scanner (Bashkirov et al 2016, Johnson et al 2016, Giacometti et al 2017b) which was validated against experimental pCT data in terms of noise and RSP accuracy (Giacometti et al 2017b, Dedes et al 2019, Dickmann et al 2019). The simulation includes the modelling of light quenching within the energy detector of the pCT scanner based on experimental data (Dickmann et al 2019). The same (Birks 1951) reference parameters were used in this work as in Dickmann et al (2019).

The scanner itself consists of two silicon strip tracking detectors which measure position and direction of each particle before and after traversing a phantom placed in between the trackers. The distance between the inner sides of the housing of the two tracking detectors is $284 \mathrm{~mm}$, which corresponds to a distance of $\pm 142 \mathrm{~mm}$ from the isocenter. Afterwards, a five-stage scintillating energy detector measures the energy deposit of the particles in each stage and converts the reading to WEPL following a specific calibration procedure (Bashkirov et al 2016). The simulation additionally allows placing a virtual front and rear detector between the corresponding realistic tracker plane and the phantom for so-called ideal energy, position and direction measurements which contain only statistical fluctuations from multiple Coulomb scattering and energy 
(a) resolution phantom

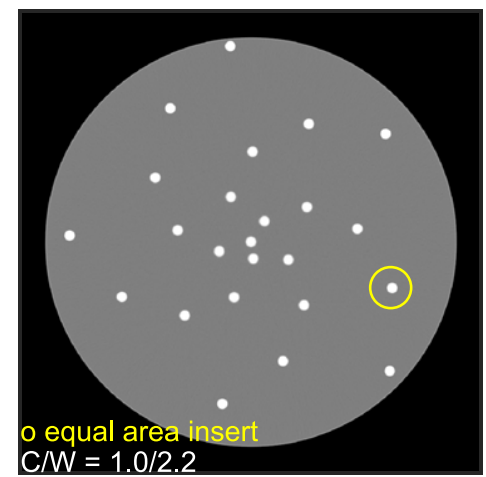

(d) head phantom

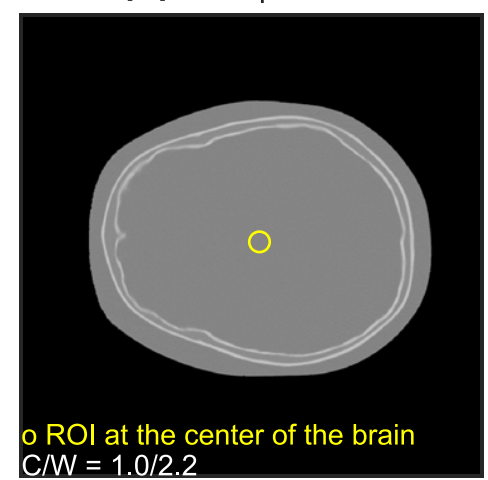

(b) water phantom

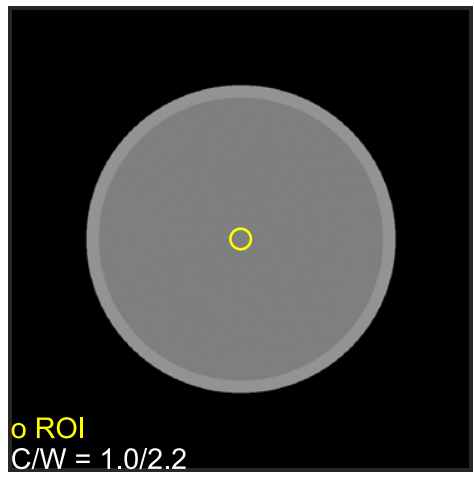

(e) sensitometry phantom

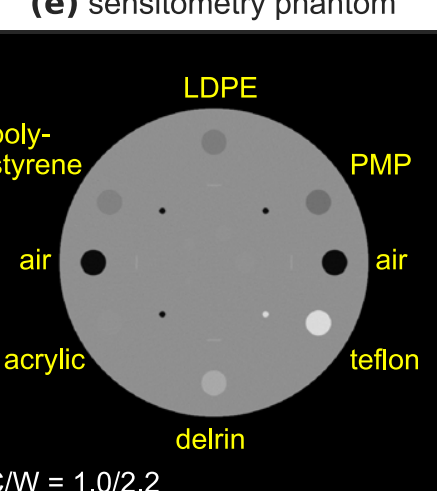

(c) head phantom

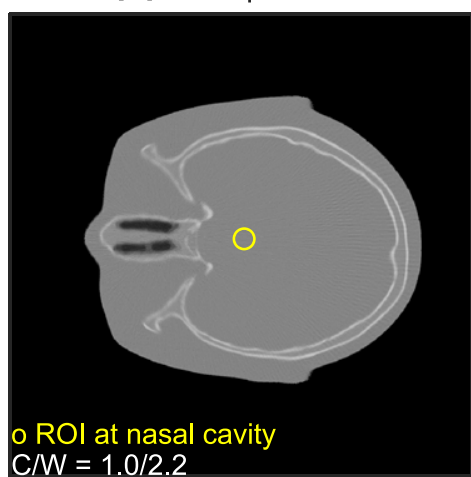

$\mathrm{C} / \mathrm{W}=1.0 / 2.2$

Figure 1. RSP maps of phantoms that were investigated in this study: (a) resolution phantom with marked equal-area insert, (b) water and ((c), (d)) head phantom with corresponding ROIs, and (e) sensitometry phantom with insert material. The RSP maps are reconstructed from the ideal dataset of the HeCT simulation.

straggling of the particles and are not modified by the uncertainties originating from the tracking process and the WEPL determination procedure. Both ideal tracker planes were located at the inner sides of the housing of the two realistic tracking detectors at a distance of $\pm 142 \mathrm{~mm}$ from the isocenter.

To calibrate the five-stage scintillating detector's energy scale in real-world acquisitions, the response of each analog-to-digital converter (ADC) in the empty calibration run is mapped to pre-calculated energy deposits $E_{n}^{\mathrm{G} 4}$ which allows to account for spatial variations in the converter signals (Bashkirov et al 2016, Dickmann et al 2019). For protons, we used the same $E_{n}^{\mathrm{G} 4}$ values as in previous studies (Bashkirov et al 2016, Dickmann et al 2019) while for helium, the values were analogously determined for each stage $n$ in an additional simulation without quenching or object, yielding $E_{n}^{\mathrm{G} 4, \mathrm{He}}=\{100.97,112.21,130.45,168.87,271.10\} \mathrm{MeV}$. In simulated acquisitions, the energy deposited in the five-stage detector is first converted to ADC including their spatial variation (see section 6.2 of Dedes et al (2020)).

We differentiate between two types of datasets_-an ideal one which refers to the data obtained by the virtual tracking detector, and a realistic one that refers to the data measured using the silicon strip trackers and the five stage energy detector. Using the event ID of each simulated particle, which is identical for both datasets, the ideal and the realistic data were further merged to a third semi-realistic dataset which consists of ideal energy values but realistic tracking positions and directions. This dataset was used to investigate the impact of measurement errors of the tracking detector on spatial resolution.

For our study, we simulated scans of each phantom with a $200 \mathrm{MeV}$ proton and $800 \mathrm{MeV}$ helium parallel beam which have about the same range. The phantoms which were used are described in section 2.2. The fluence of the beam was 417 particles $\cdot$ projection ${ }^{-1} \cdot \mathrm{mm}^{-2}$ in case of the water and the resolution phantom and 104 particles projection ${ }^{-1} \cdot \mathrm{mm}^{-2}$ for the head and the sensitometry phantom with the particles distributed uniformly across the beam. The beam size was $240 \times 40 \mathrm{~mm}^{2}$ for all phantoms except for the head phantom for which the beam was enlarged to $250 \times 70 \mathrm{~mm}^{2}$. Each simulation consisted of 360 projections in $1^{\circ}$ angle steps using a step-and-shoot mode.

\subsection{Phantoms}

Five phantoms were used in this study: a calibration phantom, a resolution phantom, a water phantom, a head phantom and a sensitometry phantom. The RSP maps of the last four phantoms as well as specific region-ofinterest (ROI)s, whose use is described in detail in section 2.4, are shown in figure 1. 
The calibration phantom (Piersimoni et al 2017) was used to simulate the calibration runs of the pCT scanner. It consists of two polysterene wedges of varying thickness between 0 and $50.8 \mathrm{~mm}$. Up to four additional bricks of $50.8 \mathrm{~mm}$ thickness were placed behind the wedges in each calibration run to cover the whole WEPL range of the energy detector.

The resolution phantom (Rit et al 2013) was used for the analysis of spatial resolution. It consists of a $200 \mathrm{~mm}$ diameter water cylinder in which aluminium rods of $5 \mathrm{~mm}$ diameter were placed at regularly increasing distances to the phantom center. Since spatial resolution in ion CT tends to be lower at the center of the object and increases towards the edge as most likely path (MLP) errors decrease, we selected the insert closest to the radius splitting the phantom in a disk and annulus of equal area as reference position for evaluations of the spatial resolution of protons and helium ions. This insert, which we call equal-area insert within this paper, is placed at a radius of $r=72 \mathrm{~mm}$. Outer inserts yield similar maximum spatial resolution for protons and helium ions due to the convergence of the MLP error to 0 at the phantom edge, while inner inserts have lowest resolution. The choice of the equal-area insert is seen as a compromise between these extrema.

The water phantom is made of a water cylinder of $150.5 \mathrm{~mm}$ outer diameter including a PMMA shell of $6.35 \mathrm{~mm}$ wall thickness. The head phantom (Giacometti et al 2017a) consists of the voxelised representation of the paediatric head phantom $\left(\right.$ ATOM $^{\circledR}$, Model 715 HN, CIRS Inc., Norfolk, VA) which was created from high quality $\mathrm{x}$-ray CT scans of the physical phantom. This phantom models the head of a 5 year old child using tissueequivalent materials. Specific ROIs of the water phantom and the head phantom (figure 1) were used for the investigation of dose deposition and image variance.

Finally, the sensitometric phantom which represents an analytical model of the CTP404 phantom (The Phantom Laboratory, New York, USA) was used for comparing the accuracy of the RSP values between pCT and HeCT. It consists of a cylindrical epoxy body of $150 \mathrm{~mm}$ diameter and eight inserts of $12.2 \mathrm{~mm}$ diameter of which two are filled with air and the remaining with tissue-equivalent materials.

Except for the head phantom, all phantoms were analytical models. The RSP, density and composition of each phantom in the simulation was the same as in Dickmann et al (2019), as was the I-value of water (78.0 eV). The sensitometry phantom's ground truth RSP was calculated for a proton energy of $150 \mathrm{MeV}$.

\subsection{Calibration and reconstruction}

Prior to image reconstruction, additional calibration runs using the calibration phantom (section 2.2) were simulated for both protons at $200 \mathrm{MeV}$ and helium ions at $800 \mathrm{MeV}$ energy. The calibration beams had parallel beam geometry with a dimension of $80 \mathrm{~mm} \times 400 \mathrm{~mm}$, which covers the entire detector surface. The fluence of each calibration run was 313 particles $\cdot \mathrm{mm}^{-2}$ within each of the 5 steps of the calibration run. In each additional step after the empty calibration run first the wedge and then the additional bricks are added. Using the path information from the trackers and the known RSP of the calibration phantom, the data of this simulation was used to create a look-up table for the conversion of the detector response signal to the corresponding WEPL values (Bashkirov et al 2016) during the preprocessing of the simulated data. To reduce the distortion of measurements by secondary fragments in HeCT, we additionally implemented a $\Delta E-E$ filter (Volz et al 2018), which allows the distinction of helium ions from their fragments by comparing the energy deposit in the previous stage $E_{\Delta E}$ of the energy detector to the energy deposit of the stopping stage $E_{\mathrm{BP}}$. More precisely, we determined two boundaries for the signal of the energy detector, a higher and a lower one as seen in figure 4(b) of Volz et al (2018), outside which particles are rejected. These boundaries were parameterised according to

$$
E_{B P}=a E_{\Delta E}^{2}+b E_{\Delta E}+c
$$

with parameters $a, b$ and $c$ which are shown in the appendix (table A.1). The parameters for stage 3 were extracted from the $\Delta E-E$ filter boundaries in figure 4(b) of Volz et al (2018) which apply to helium beam measurements and matched the data of our simulation well. For the other stages, these parameters needed to be slightly adapted to the stage signal (data not available in Volz et al 2018). When using the ideal data of the virtual detector for image reconstruction, the entire calibration and preprocessing procedure is skipped since the ideal measurements can be directly used for the image reconstruction (after applying $3 \sigma$ rejection explained below).

After preprocessing, both particle types were grouped on a $2 \mathrm{~mm} \times 2 \mathrm{~mm}$ binning grid according to their front tracker coordinate and particles outside of $3 \sigma$ angle and WEPL around the respective mean were rejected (Schulte et al 2005) where $1 \sigma$ corresponds to the standard deviation. For the remaining particles, the MLP was calculated according to Schulte et al (2008) to create 3D projections at binning sizes of $0.1 \mathrm{~mm} \times 3 \mathrm{~mm} \times 1 \mathrm{~mm}$ and $0.25 \mathrm{~mm} \times 3 \mathrm{~mm} \times 1 \mathrm{~mm}$ in the $u, v$ and $d$ directions. The direction $d$ was parallel to the ion beam, $v$ parallel to the rotation axis and $u$ normal to both.

DDB as part of the FBP reconstruction algorithm allows to select the WEPL value of the optimal binning depth to the corresponding voxel position (Rit et al 2013). The fit parameters required for the estimation of the MLP of helium ions were estimated as in Schulte et al (2008) (equation (29) in that paper). A water volume (Ivalue $78.0 \mathrm{eV}$ and density $1 \mathrm{~g} \mathrm{~cm}^{-3}$ ) of $20 \mathrm{~cm}$ was used to score the energy of $200 \mathrm{MeV}$ protons and $800 \mathrm{MeV}$ 
helium ions as a function of depth in $5 \mathrm{~mm}$ steps (see equation (28) in Schulte et al 2008). We confirmed that for protons we obtained parameters agreeing within $1 \%$ of Schulte et al (2008). The helium parameters are reported in the appendix (table A.2).

Prior to back projection, we applied a Hann window to the ramp filter of the filtered backprojection algorithm, i.e. in Fourier space

$$
G_{\text {Hann }}(q)=|q| \operatorname{rect}\left(\frac{q}{W_{\text {Hann }}}\right)\left[0.5+0.5 \cos \left(\frac{\pi q}{W_{\text {Hann }}}\right)\right],
$$

where $|q|$ is the Fourier transform of the ramp filter of frequency $q$ normalised by the Nyquist-Shannon frequency. The apodized Hann filter $G_{\text {Hann }}$ in frequency space was used to modify image quality in terms of noise and spatial resolution by reducing the effect of the high frequency amplification of the ramp filter. Different cutoff frequencies $W_{\text {Hann }}$ ranging from 0 to 1 can be chosen to modify $G_{\text {Hann }}$ and therefore the amount of smoothing which increases with decreasing values of $W_{\mathrm{Hann}}$. The application of Hann windowing is the same as using reconstruction kernels in $\mathrm{x}$-ray CT.

The fully reconstructed RSP maps consisted of either $0.1 \mathrm{~mm}$ or $0.25 \mathrm{~mm}$ pixel size and $3 \mathrm{~mm}$ slice thickness. If not mentioned otherwise, the larger pixels size of $0.25 \mathrm{~mm}$ was used. We applied the small pixel size in case of the spatial resolution analysis to both ideal and realistic data in section 3.1 to evaluate the impact of the pixel size on ideal spatial resolution.

Along with the image reconstruction, we used the variance reconstruction of Rädler et al (2018), which was validated in Dickmann et al (2019), to create the corresponding variance maps of the reconstructed images from the variance of the projection values. It was necessary to adapt the variance reconstruction formalism to account for the effect of the Hann filter since Rädler et al (2018) accounted for the ramp filter only. This is presented in the appendix and variance was reconstructed using equation (A.11), which we implemented in RTK similarly as in Dickmann et al (2019).

\subsection{Data evaluation}

The performance of pCT and HeCT was investigated using four different parameters: spatial resolution, variance, dose and RSP accuracy.

The spatial resolution was evaluated by calculating the modulation transfer function (MTF) from the radial edge-spread function (ESF) (Richard et al 2012) of the aluminium inserts using the central slice of the resolution phantom. We followed the approach of Krah et al (2018) and Khellaf et al (2020) and modelled the ESF as an error function with parameters $A, \mu, \sigma$ and $C$

$$
\operatorname{ESF}(r)=\frac{A}{2}\left(1+\operatorname{erf}\left(\frac{r-\mu}{\sqrt{2} \sigma}\right)\right)+C,
$$

where $r$ is the distance of the sampled points to the insert center. The spatial frequency of the MTF at the $10 \%$ level can be derived from $\sigma$ according to

$$
f_{\mathrm{MTF}_{10 \%}}=\frac{1}{\pi \sigma} \cdot \sqrt{\frac{\ln 10}{2}}
$$

and is used as a measure of the spatial resolution within this study. As already described in section 2.2, the equalarea insert of the resolution phantom $(r=72 \mathrm{~mm})$ was chosen as a reference position for some evaluations of the spatial resolution due to the dependence of the spatial resolution analysis on the insert position within the phantom.

The variance of proton and helium ion images was estimated using the variance reconstruction maps of the water phantom and the head phantom. As a reference, we chose the mean variance in a ROI of $1 \mathrm{~cm}$ diameter at three different positions: at the center of the water phantom and for the head phantom at the center of the brain and at the nasal cavity. We chose homogeneous and heterogeneous phantoms due to the increase of noise around heterogeneities caused by multiple Coulomb scattering (Dickmann et al 2019).

The dose was obtained by scoring the absorbed doses from the Monte Carlo simulation for each projection separately on a $2 \times 2 \times 2 \mathrm{~mm}^{3}$ dose grid. Summing over all projections gave the final dose. We also determined the average dose value within the same ROIs that were selected for the variance for both water and head phantom.

To further compare variance and dose in both proton and helium reconstructions, we made use of the following relation

$$
V \cdot D=\text { constant }
$$




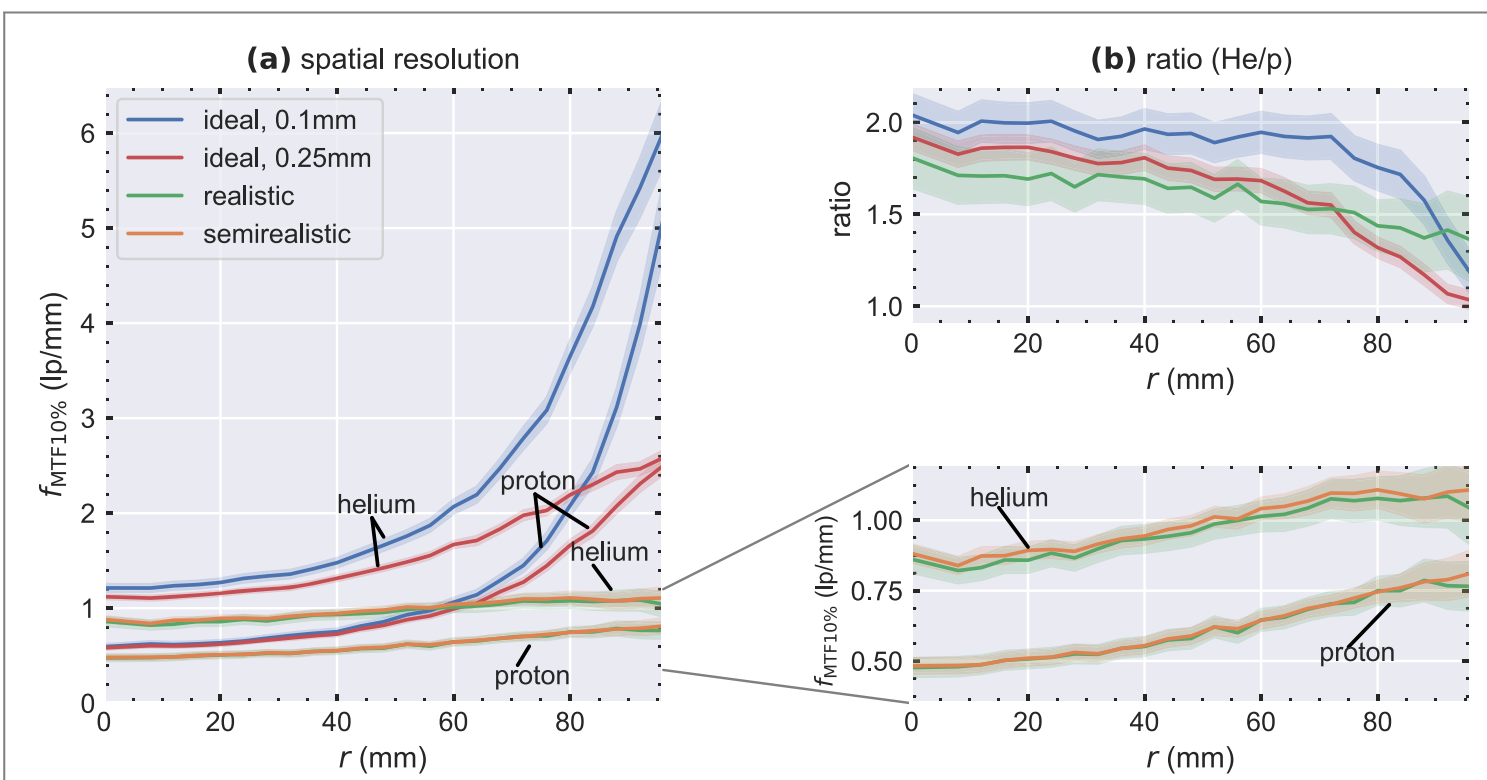

Figure 2. (a) Spatial resolution of protons and helium ions for the ideal, realistic and semi-realisitic dataset which were reconstructed without Hann filtering. (b) Ratio of the spatial resolution of helium ions to protons for the ideal and realistic dataset. The shaded area displays in all graphs a $3 \sigma$ uncertainty on the spatial frequency $f_{\mathrm{MTF}_{10 \%}}$.

to re-scale image variance $V$ and dose $D$ obtained from one simulation and its reconstructions. The relation is valid with an uncertainty below 5\% if the projection bins have at least 30 counts (see figure 4 in Dickmann et al 2020a), which we ensured was the case.

The RSP accuracy was similarly evaluated as in Dickmann et al (2021c) using the central slice of the sensitometry phantom. We calculated the $\mathrm{RSP}_{\text {mean }}$ within a radius of $3 \mathrm{~mm}$ towards each insert center for the inserts and $10 \mathrm{~mm}$ towards the phantom center for the body which we compared to the reference RSP ref $_{(}$(which corresponds to the phantom's ground truth described in section 2.2) using a relative error $\left(\mathrm{RSP}_{\text {mean }}-\mathrm{RSP}_{\mathrm{ref}}\right) / \mathrm{RSP}_{\text {ref. }}$ The The mean absolute percentage error (MAPE) was afterwards calculated from the $M$ inserts as

$$
\mathrm{MAPE}=\frac{100 \%}{M} \sum_{m=1}^{M} \frac{\left|\mathrm{RSP}_{\text {mean }, m}-\mathrm{RSP}_{\mathrm{ref}, m}\right|}{\mathrm{RSP}_{\mathrm{ref}, m}} .
$$

\subsection{Spatial resolution matching}

For the final comparison of protons and helium ions, the goal was to fix two of the three parameters dose, variance (which are strictly linked) and spatial resolution and use the third parameter for comparison. For this purpose, we first adjusted the spatial resolution of both particles at the equal-area insert by using the Hann filter which yielded a specific image variance for both particles. Specifically, helium spatial resolution was reduced to the spatial resolution of protons at $W_{\mathrm{Hann}}=1$. We chose to apply the Hann filter to protons to compensate for the strongly increased variance of the pCT images caused by the small pixel size. Either dose or variance was then determined using relation (5). The reference dose was $5 \mathrm{mGy}$, while the reference variance was the proton variance at the central ROI of the water phantom simulation at the reference dose and $W_{\text {Hann }}=1$.

\section{Results}

\subsection{Spatial resolution}

Figure 2 compares the spatial resolution of protons and helium ions obtained from the reconstruction of the ideal, semi-realistic and realistic datasets of the resolution phantom. The Hann filter was not applied to the reconstruction and a pixel size of $0.1 \mathrm{~mm}$ was used (and additionally $0.25 \mathrm{~mm}$ to study pixel size effects on the ideal data). In all cases, the resolution was lowest at the phantom center and highest at the edge of the phantom. Since MLP errors vanish at the phantom's surface, at the largest radius the ideal results for both ions converged within uncertainty to a limit governed by the pixel size. This can be appreciated when comparing ideal results with 0.1 and $0.25 \mathrm{~mm}$ pixel sizes. For the $0.1 \mathrm{~mm}$ pixel size, spatial resolution at the center was found to be $0.59 \mathrm{lp} \mathrm{mm}^{-1}$ for protons and $1.21 \mathrm{lp} \mathrm{mm}^{-1}$ for helium in the ideal case, and 0.48 and $0.86 \mathrm{lp} \mathrm{mm}^{-1}$ in the realistic case. The resolution ratio of helium ions to protons in the ideal $0.1 \mathrm{~mm}$ pixel size case was found to be 

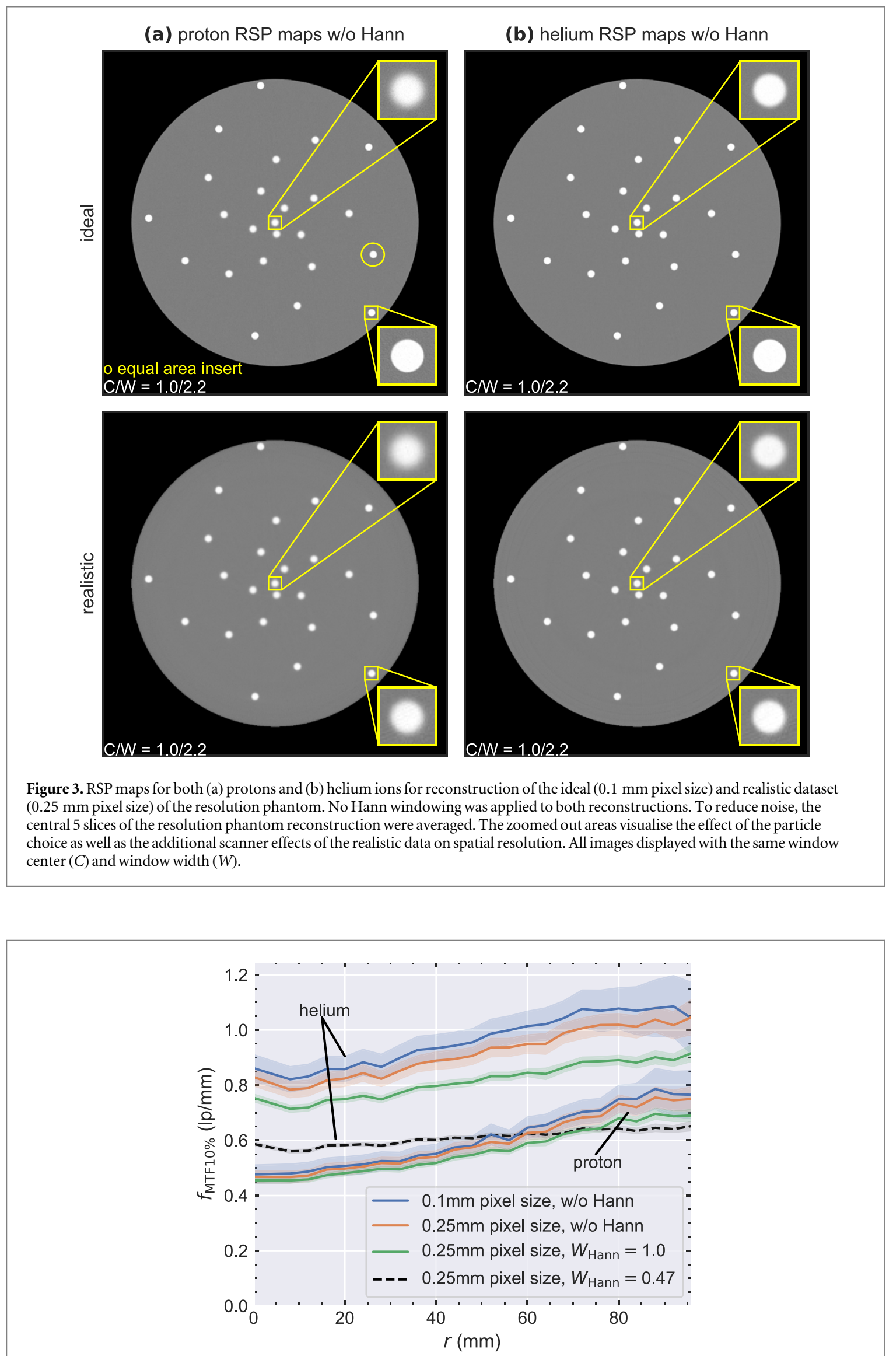

Figure 4. Decrease of the spatial resolution in realistic pCT and HeCT images by increasing the pixel size from 0.1 to $0.25 \mathrm{~mm}$ and additional application of the Hann filter at $W_{\mathrm{Hann}}=1$. The black dashed line additionally displays the spatial resolution of helium ions at $W_{\mathrm{Hann}}=0.47$ as required for the resolution matching of section 3.4. 


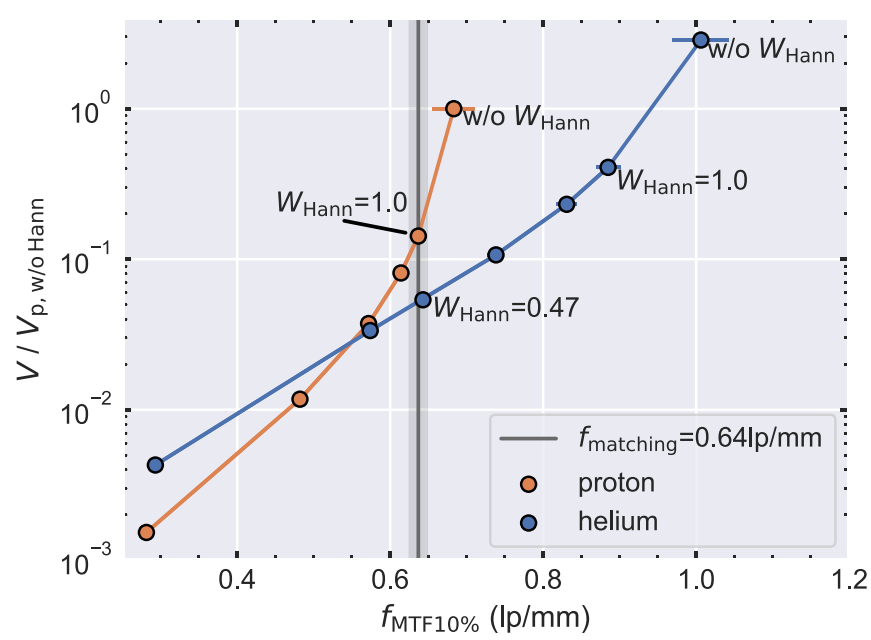

Figure 5. Variance at the central ROI of the water phantom for both ions normalized to the proton variance without Hann windowing $\left(V_{p, \text { w } / \mathrm{o} \text { Hann }}=0.0022\right)$. The normalized variance is plotted versus spatial resolution of the equal-area insert of the resolution phantom $(r=72 \mathrm{~mm})$ for each particle. The different resolutions and variances are obtained from applying different $W_{\text {Hann }}($ from left to right: $0.2,0.4,0.6,0.8,1$ and without). For helium, an additional point is shown for $W_{\mathrm{Hann}}=0.47$ which matches the proton spatial resolution as indicated by the gray vertical bar (shaded area/error bars show a $3 \sigma$ uncertainty). Dose was rescaled to $5 \mathrm{mGy}$ for both particles according to equation (5).

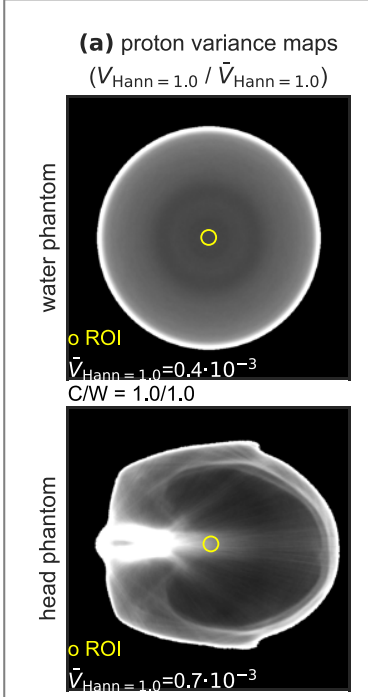

(b) helium variance maps
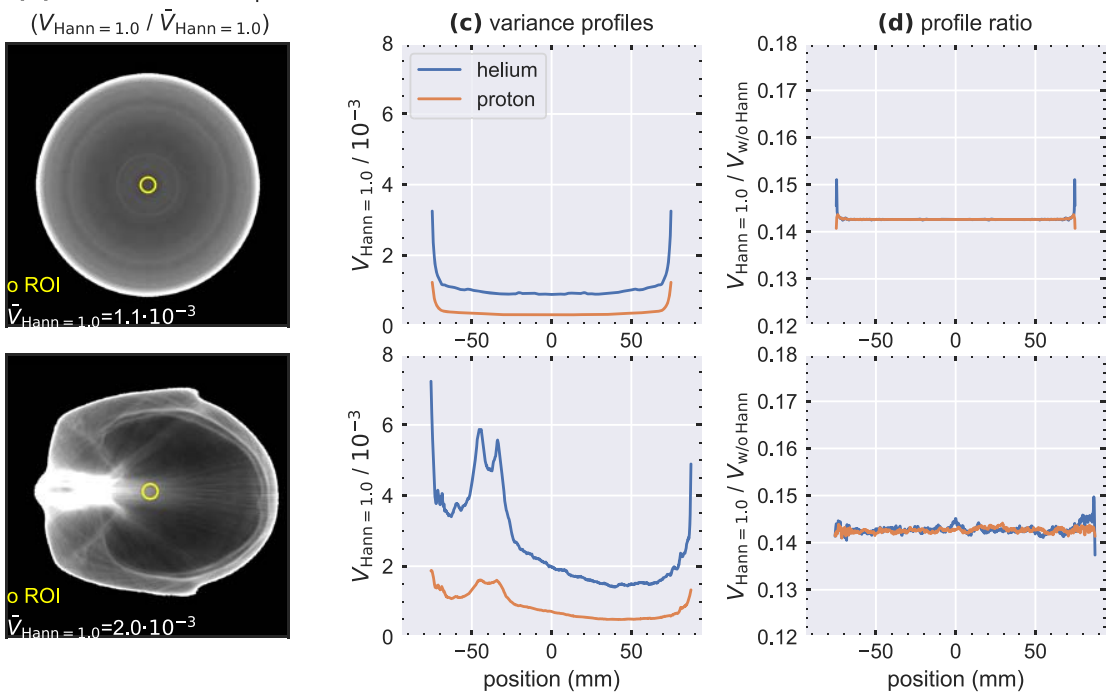

Figure 6. Variance maps of the water phantom and the head phantom for protons (a) and helium ions (b) at a dose of $5 \mathrm{mGy}$ in each ROI as well as corresponding variance profiles (c) at $W_{\text {Hann }}=1$. (d) Displays the variance profile ratio of the phantoms with applied Hann filter to the profile without Hann filter $V_{\mathrm{Hann}=1} / V_{\mathrm{w} / \mathrm{o}}$ Hann. The profiles as well as the profile ratio were determined after applying an image mask to the variance maps. The variance maps are normalised to their overall mean variance $\bar{V}_{\text {Hann }=1}$. The yellow circle indicates the ROI that was used for dose and variance measurements. Center and window $C / W$ applies to all variance maps.

2.0 at the center of the resolution phantom, and approaches unity towards the edge of the phantom. The ratios of the realistic dataset were 1.8 at the center and 1.4 at the edge. The semirealistic dataset exhibited good agreement with the resolution of the realistic dataset, confirming the expectation that the tracking detectors govern the resolution performance of the scanner (Krah et al 2018, Khellaf et al 2020).

The visual difference between the reconstructions of the ideal and realistic datasets of the resolution phantom is shown in figure 3 for both (a) protons and (b) helium ions. It is clearly visible that central inserts are more blurred than outer inserts, the change from ideal to realistic data leads to further blurring, most noticeable at the phantom's periphery, and HeCT is sharper.

The effect of the increase of the pixel size from 0.1 to $0.25 \mathrm{~mm}$ as well as the application of the additional Hann filter at full window width $\left(W_{\text {Hann }}=1\right)$ on the spatial resolution of the realistic data is displayed in figure 4 . Both changes caused a slight loss of spatial resolution over the whole phantom compared to the parameters used for figure 2. For protons, the effect was found to be small: the pixel size decreases the central spatial resolution by 
(a) proton dose maps $\left(D / \bar{D}_{\mathrm{ROI}}\right)$

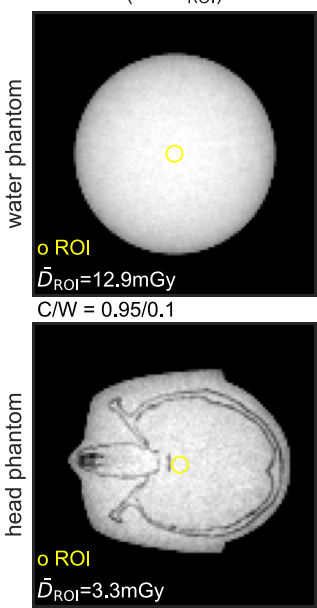

(b) helium dose maps $\left(D / \bar{D}_{\mathrm{ROI}}\right)$
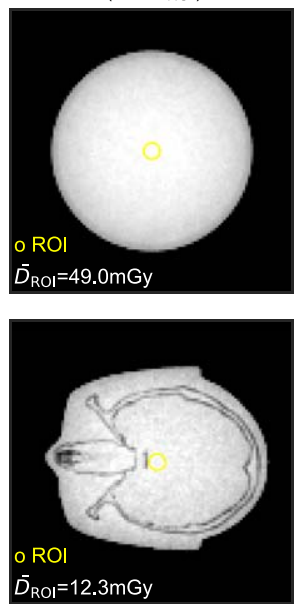

(c) dose profiles

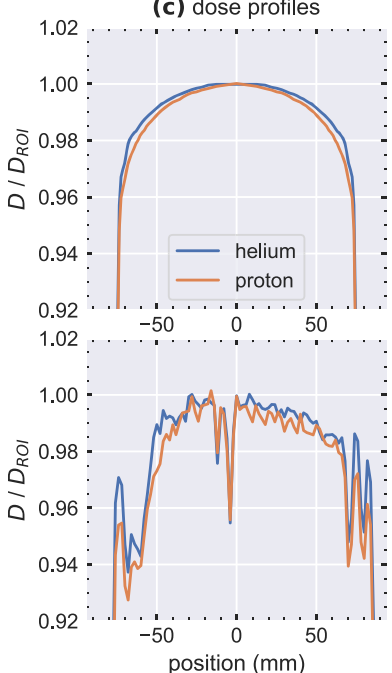

(d) dose ratio $(\mathrm{He} / \mathrm{p})$

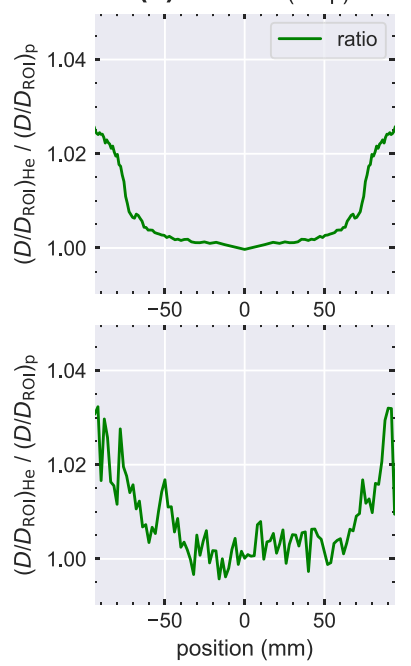

Figure 7. Dose maps of the water phantom and the head phantom for (a) protons and (b) helium ions normalized to the average dose within the central ROIs and (c) corresponding dose profiles and (d) dose profile ratios of the normalized dose profiles. The yellow circle indicates the ROI that was used for dose and variance measurements. Center and window $C / W$ applies to all dose maps. The dose maps and profiles correspond to the variance maps and profiles of figure 6 whose variance was calculated for $D_{\mathrm{ROI}}=5 \mathrm{mGy}$.

about $2 \%$ and the Hann filter additionally by $3 \%$. The central spatial resolution loss of helium ions however was $4 \%$ for the pixel size and $9 \%$ additionally for the Hann filter.

\subsection{Variance versus spatial resolution}

The relation between water phantom central ROI variance at a fixed dose (set to $5 \mathrm{mGy}$ ) and spatial resolution is shown in figure 5 where we generated a set of reconstructions from the realistic dataset of the water and the resolution phantom, each at a different $W_{\text {Hann }}$. We can appreciate that without Hann windowing, helium has both higher spatial resolution and variance than protons. At equal resolution, and above approximately $0.6 \mathrm{lp}$ $\mathrm{mm}^{-1}$, helium yields better variance than protons. The ratio of spatial resolution of helium ions to protons at the equal-area insert was 1.5 when no Hann filter was applied and 1.4 for $W_{\text {Hann }}=1$. For $W_{\text {Hann }}=0.2$, the cutoff frequency dominates spatial resolution, leading to the same resolution for both ions. Without Hann windowing, the variance of helium was 2.9 times that of protons, while at matched spatial resolution of $0.64 \mathrm{lp} \mathrm{mm}^{-1}$, it was 0.38 times that of protons.

\subsection{Image variance and image dose distribution}

Variance maps of the water phantom and the nasal cavity of the head phantom are shown in figure 6(a) for protons and figure 6(b) for helium ions. Figure 6(c) shows variance profiles and figure 6(d) the ratio of the variance profiles with and without Hann filtering. For the water phantom, the variance was smallest at the center and increased towards the edge whereas for the head phantom, the variance was elevated near heterogeneities such as the nasal cavity. The average variance ratio of helium ions to protons was 2.8 for the water and 3.0 for the head phantom. The ratios in figure 6(d) show that the Hann filter decreased variance by the same factor in each image pixel, with no spatial variation observed. The decrease was by a factor of 7 in each image pixel for both ions when applying the Hann filter at $W_{\text {Hann }}=1$ compared to no Hann filter.

The corresponding dose maps and dose profiles to figure 6 are presented in figure 7 . The dose map of the water phantom and the nasal cavity of the head phantom is shown for protons in figure 7(a) and for helium ions in figure 7(b) as well as the corresponding dose profiles in figure 7(c) where dose maps as well as the profiles are normalized to the mean dose within the chosen ROI of the phantoms. The dose decreases by about $2.5 \%$ and $3 \%$ for helium ions and protons respectively from the center of the water phantom to the periphery $(r=70 \mathrm{~mm})$ which results in an increase of the dose ratio below $1 \%$ towards the water phantom edge. The dose along the nasal cavity profile of the head phantom compared to the mean dose within the selected ROI decreases by up to $6 \%$ (helium) and 7\% (protons) towards the phantom edge. The increase of the dose ratio is below $2 \%$.

Table 1 shows the dose ratio at equal variance within the ROIs for ideal and realistic datasets. The ratio is defined as the helium dose divided by the proton dose required to reach the same variance in the ROI in a given phantom. The ideal dose ratio of helium ions to protons was found to be 1.3 for the water phantom and the center of the brain of the head phantom, and 2.1 for the head phantom's nasal cavity. For realistic data, the dose 


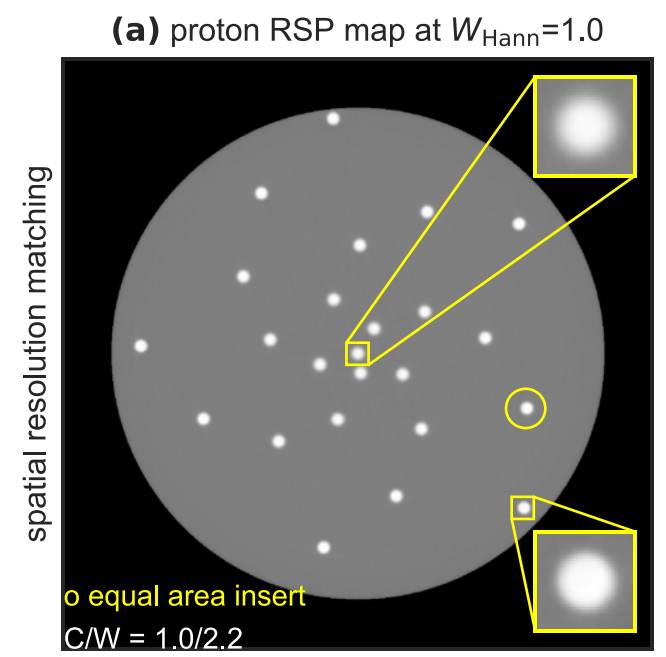

\section{(b) helium RSP map at $W_{\text {Hann }}=0.47$}

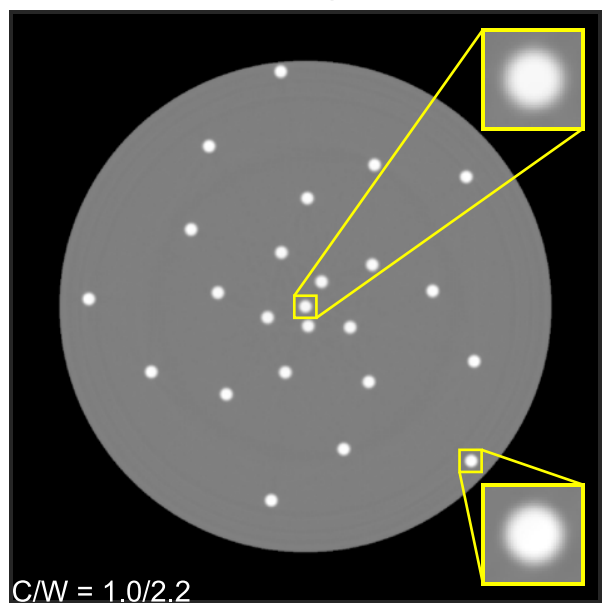

Figure 8. RSP maps for (a) protons and (b) helium ions for reconstruction of the resolution matched realistic dataset ( $0.25 \mathrm{~mm}$ pixel size) of the resolution phantom. To reduce noise the central 5 slices were averaged. Both images displaced with the same window center $(C)$ and window width $(W)$.

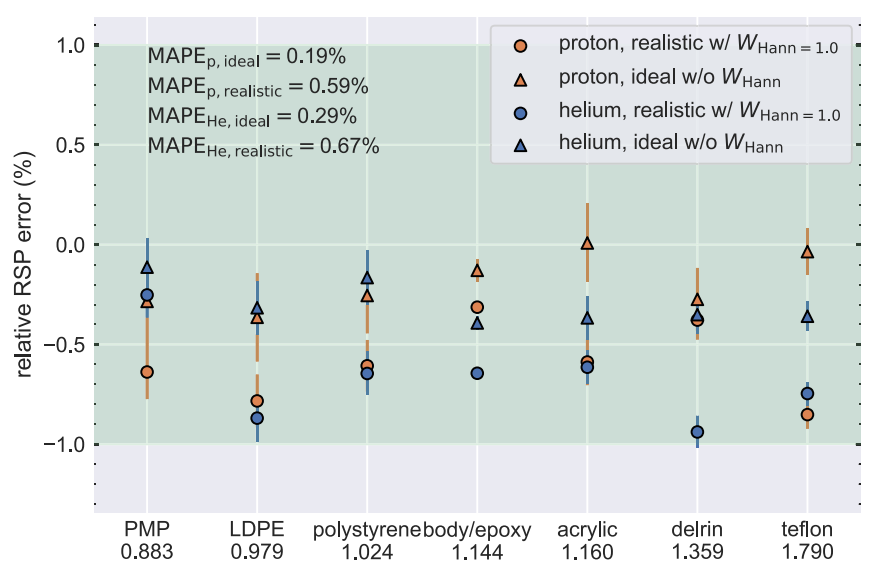

Figure 9. Relative RSP error for the different insert materials of the sensitometry phantom. The RSP value is given below each material name. The shaded area indicates a $\pm 1 \%$ range on the relative RSP accuracy. The error bar indicates a $1 \sigma$ uncertainty, where $\sigma$ is the standard error of the mean, on the obtained RSP values which is lower for the realistic data due to the applied Hann filter.

Table 1. The first three columns report the dose ratio of helium ions to protons at equal variance at the central ROI of the water phantom and for the two ROIs of the head phantom (nasal cavity and center of brain). The ratio is taken using helium and proton data from the same phantom using reconstructions without Hann windowing. The last two columns report the doses for realistic simulations required to achieve the reference variance at the center of the water cylinder for protons without Hann windowing $\left(V_{\mathrm{p}, \mathrm{w} / \mathrm{oHann}}=0.0022\right)$.

\begin{tabular}{lccccc}
\hline ROI & Ideal & $\begin{array}{c}\text { Rrealistic } \\
\text { w/o } \Delta E-E\end{array}$ & $\begin{array}{c}\text { Realistic } \\
\text { w/ } \Delta E-E\end{array}$ & $\begin{array}{c}\text { pCT dose } \\
(\mathrm{mGy})\end{array}$ & $\begin{array}{c}\text { HeCT dose w/ } \Delta E-E \\
(\mathrm{mGy})\end{array}$ \\
\hline Water phantom & 1.3 & 3.7 & 2.9 & 5.00 & 14.29 \\
Nasal cavity & 2.1 & 3.8 & 2.9 & 11.29 & 32.34 \\
Center of brain & 1.3 & 3.1 & 2.8 & 5.52 & 15.31 \\
\hline
\end{tabular}

ratio of helium ions to protons was 3.7 (water phantom), 3.8 (head nasal cavity) and 3.1 (head center of brain) without the $\Delta E-E$ filter. Applying the $\Delta E-E$ filter reduces the dose ratios to 2.9, 2.9 and 2.8 respectively. Table 1 additionally reports the $\mathrm{pCT}$ and $\mathrm{HeCT}$ doses required to achieve the reference variance for each phantom in realistic simulations. We can observe that as expected for the nasal cavity ROI, more dose is required due to the increase of variance near heterogeneities. 
Table 2. Dose ratio of helium $\left(W_{\mathrm{Hann}}=0.47\right)$ to protons

$\left(W_{\text {Hann }}=1.0\right)$ at resolution matching $\left(0.64 \mathrm{lp} \cdot \mathrm{mm}^{-1}\right.$ at equal-area insert) and equal variance. The last two columns report the doses for realistic simulations required to achieve the reference variance at the center of the water cylinder for protons $\left(V_{\mathrm{p}, \mathrm{W}_{\text {Hann }}=1.0}=0.00031\right)$.

\begin{tabular}{lccc}
\hline ROI & $\begin{array}{c}\text { Dose ratio } \\
(\mathrm{He} / \mathrm{p})\end{array}$ & $\begin{array}{c}\text { pCT dose } \\
(\mathrm{mGy})\end{array}$ & $\begin{array}{c}\text { HeCT dose } \\
(\mathrm{mGy})\end{array}$ \\
\hline Water phantom & 0.38 & 5.00 & 1.89 \\
Nasal cavity & 0.38 & 11.28 & 4.24 \\
Center of brain & 0.36 & 5.52 & 2.01
\end{tabular}

\subsection{Spatial resolution matching.}

At the equal-area insert $(r=72 \mathrm{~mm})$, protons had a spatial resolution of $0.64 \mathrm{lp} \cdot \mathrm{mm}^{-1}$ with $W_{\text {Hann }}=1$. This was matched with helium ions by using $W_{\text {Hann }}=0.47$. In that case, helium ions still had a higher resolution at the phantom center $\left(0.59 \mathrm{lp} \cdot \mathrm{mm}^{-1}\right.$ compared to $0.45 \mathrm{lp} \cdot \mathrm{mm}^{-1}$ for protons, see figure 4$)$ but a lower resolution at the phantom periphery $\left(0.64 \mathrm{lp} \cdot \mathrm{mm}^{-1}\right.$ compared to $0.69 \mathrm{lp} \cdot \mathrm{mm}^{-1}$ for protons). The resolution matched reconstructions of the resolution phantom for $\mathrm{pCT}$ and $\mathrm{HeCT}$ are shown in figure 8.

The results for the dose ratio at resolution matching of helium ions to protons at equal variance is shown in table 2. For all three ROIs, the dose ratio decreased in favour of helium ions to 0.38 for the water phantom and the head phantom's nasal cavity ROI and to 0.36 for the center of the brain of the head phantom's ROI. Table 2 additionally reports the $\mathrm{pCT}$ and $\mathrm{HeCT}$ doses required to achieve the reference variance for each phantom in realistic simulations.

\subsection{RSP accuracy.}

The RSP accuracy is shown for protons and helium ions in figure 9. Both particles slightly underestimate the actual RSP value within a relative error range of $1 \%$. Helium shows a slightly worse accuracy of $\mathrm{MAPE}_{\mathrm{He}}=0.29 \%$ than protons with $\mathrm{MAPE}_{\mathrm{p}}=0.19 \%$ for the ideal dataset. For the realistic dataset, we have $\mathrm{MAPE}_{\mathrm{He}}=0.67 \%$ and $\mathrm{MAPE}_{\mathrm{p}}=0.59 \%$.

\section{Discussion}

The analysis of the two different types of datasets allows disentangling uncertainty contributions from the measurement processes and from statistical limitations of particle imaging. Specifically, we found that the uncertainty of the position and direction measurements, which is higher for the semirealistic and realistic datasets than for the ideal dataset, contributes importantly to the achievable spatial resolution as expected from Krah et al (2018) and Khellaf et al (2020). This effect is more prevalent towards the resolution phantom's periphery while at the center the effect is more pronounced for helium ions than for protons.

The spatial resolution as represented by the spatial frequency of the MTF at the $10 \%$ level is inversely proportional to the paramter $\sigma$ of the error function fit (see equation (4)). This parameter is dominated to the uncertainty of the MLP $\sigma_{\mathrm{MLP}}$ (Krah et al 2018) which originates from the scattering of the particles (equation (9)-(11) of Krah et al 2018 or, equation (7)-(9) and equation (16)-(18) of Schulte et al 2008) as described by Highland's formula (equation (28) and (39) in Gottschalk et al 1993) which is proportional to the particle's charge and inversely proportional to the particle's mass (as given by the momentum in the Highland's formula) for equal particle velocity. This applies over the whole phantom range in our case due to the same initial specific energy of the protons and helium ions. Since the helium ion mass is 4 times that of the proton mass and the particle charge is 2 times that of the proton charge, the spatial resolution is expected to scale by a factor of 2 , which agrees with the central spatial resolution ratio of the ideal data in figure 2(b) but is slightly reduced for the realistic data to 1.8 .

The above observations further agree well with the model introduced by Collins-Fekete et al (2021) who determined a limiting spatial resolution introduced by multiple Coulomb scattering (MCS) of $0.57 \mathrm{lp} \mathrm{mm}^{-1}$ for protons and about $1.15 \mathrm{lp} \mathrm{mm}^{-1}$ for helium ions at an energy of $200 \mathrm{MeV} / \mathrm{u}$ when crossing $20 \mathrm{~cm}$ of water (see figure 3 of Collins-Fekete et al 2021). This corresponds to the central spatial resolution values of the resolution phantom when reconstructing the ideal dataset (see figure 2, $0.59 \mathrm{lp} \mathrm{mm}^{-1}$ for pCT and $1.21 \mathrm{lp} \mathrm{mm}^{-1}$ for $\mathrm{HeCT}$ ). For the reconstruction of the realistic dataset, our values deviated partially from Collins-Fekete et al (2021), namely by 0.096 and $0.29 \mathrm{lp} \mathrm{mm}^{-1}$ for protons and helium ions respectively, probably because that study did not account for specific tracker uncertainties. However, the central spatial resolution of the HeCT acquisition, which is $0.86 \mathrm{lp} \mathrm{mm}^{-1}$ for a pixel size of $0.1 \mathrm{~mm}$ in the realistic case, is slightly higher than what was 
found experimentally by Volz et al (2021) ( $\left.0.59 \mathrm{lp} \mathrm{mm}^{-1}\right)$ which might be attributed to the larger pixel size of $0.997 \mathrm{~mm}$ for the reconstructions as well as the different phantom geometry and reconstruction method. In general, the increased resolution towards the phantom's edge is attributed to the reduced uncertainty for the particles' MLP at the periphery.

The reason for choosing small pixels for the analysis of the spatial resolution (section 3.1) was to avoid limitations on the attainable spatial resolution which might be introduced by larger pixel sizes. However, this is only relevant for reconstructions of the ideal dataset. For realistic reconstructions, the decrease of the spatial resolution over the whole phantom is low for protons when increasing the pixel size from 0.1 to $0.25 \mathrm{~mm}$. Similarly, the Hann filter leads to only minor further changes of the proton resolution. At the same time, the Hann filter helps to reduce some of the increased variance values in the pCT images that were introduced by choosing the small pixel size (see variance reduction by a factor of 7 in each image pixel with $W_{\text {Hann }}=1$ in figure $6(\mathrm{~d}))$. Therefore, the chosen parameters for the reference proton reconstructions $(0.25 \mathrm{~mm}$ pixel size and $W_{\text {Hann }}=1$ ) can be considered as a well-balanced choice of the reference parameters for the comparison of protons to helium.

The model of Collins-Fekete et al (2021), which describes the noise at the center of a water cylinder and models effects from attenuation losses of primary particles in an idealized detector, predicted a $36 \%$ dose increase at the same signal-to-noise ratio (SNR) for helium ions compared to protons. This value is close to what we obtained for the ideal dataset in case of homogeneous phantom regions, the ROI of the water phantom $(27 \%$ dose increase) and the center of the brain (33\% dose increase), at equal image variance. However, the value is different for inhomogeneous regions like the ROI of the nasal cavity ( $109 \%$ dose increase). These results are independent of the additional smoothing caused by the Hann filter since the image variance is always decreased by the same factor when $W_{\mathrm{Hann}}$ is equal for both particle types (figure 6). For the realistic datasets, the dose ratios however increase since both phantom simulations include energy straggling and nuclear interactions within the five-stage energy detector (see table 1 ). The impact of nuclear reactions of the helium ions on the dose ratio can be reduced by applying the $\Delta E-E$ filter, but the dose ratio from the ideal dataset cannot be fully recovered.

The image variance is proportional to the variance of the particle range and inversely proportional to the number of particles detected. After traversing the center of the water phantom, approximately $86 \%$ of the primary protons and $71 \%$ of the primary helium ions (figure 2.7 of Volz 2021) remain and may be used for the image reconstruction where 4 times more protons need to traverse the ROI for the same image dose. The variance of range straggling is inversely proportional to the mass at the same particle range (Durante and Paganetti 2016) and therefore for helium ions a factor of 4 less than protons. The variance ratio of helium ions to protons is therefore expected to be 1.2 in case of equal dose (or a dose increase of $21 \%$ at equal noise). This is close to the dose increase in the water phantom (27\%) and the center of the brain of the head phantom (33\%) in the ideal case but underestimates the dose increase in heterogeneous regions like the nasal cavity of the head phantom (109\%).

Since our parameters for the $\Delta E-E$ filter of stage 3 (see table A.1) agree well with the experimental data as shown in figure 4(b) of Volz et al (2018), we further think that our quenching model for the energy detector and therefore the $\Delta E-E$ filter is reasonably accurate. For the other stages, we can unfortunately not comment since these data were not published by Volz et al (2018). The HeCT RSP accuracy was approximately at the same level as the accuracy of protons with a relative error below $1 \%$ in both cases, which can be attributed to the benefits of the $\Delta E-E$ filter for Helium (Volz et al 2018).

Within the Monte Carlo simulation of the phase-II pCT scanner, there is no pile-up of particles simulated. However, our previous studies on the phase-II pCT scanner already show that the realistic data acquired from the simulation is highly representative of the real data acquired in a scan with the phase-II pCT scanner (see for example figure 6 of Dickmann et al 2019). Beam current, detector readout rate and false hits are therefore expected to not notably affect the outcome of our study.

Resolution matching is common in x-ray CT (Tilley et al 2015) and was applied to ion CT by Collins-Fekete et al (2021) by introducing a considerable increase of the proton energy to $350.0 \mathrm{MeV} / \mathrm{u}$ as well as a decrease of the helium energy to $191.1 \mathrm{MeV} / \mathrm{u}$. The results in figure 4 of Collins-Fekete et al (2021) indicate that this would lead to a dose ratio of helium ions to protons of about 0.43 . Our proposed procedure, which applies previously established energy ranges, leads to similar results with dose ratios between 0.36 and 0.38 . Figure 7 further indicates that this dose advantage is valid also for areas outside the ROI of the phantoms due to the negligible increase of the dose ratio below $2 \%$ for equal particle fluence and when the variance is equalized within the ROI.

Our study has focused on spatial resolution, image variance and physical dose as image quality parameters. For future clinical applications, the biological effect of the different particle types needs to be considered. Detailed investigations on the biological dose can be found in Hansen et al (2014) and Meyer et al (2019) who determined quality factors (see table 1 of Hansen et al 2014) and relative biological effectiveness (RBE) (see table 1 of Meyer et al 2019) for protons and helium ions. Those are almost identical for both particle types, and 
the values for helium ions deviate by less then $10 \%$ from those of protons in both publications. This would only slightly modify our final results.

Finally, pCT and HeCT show acceptable RSP accuracy with a relative RSP error below $1 \%$. This agrees with the result of Volz et al (2021) who found a MAPE of $0.30 \%$ for helium ions (values without body material) for an experimental sensitometry phantom scan which is slightly better than our value of $\mathrm{MAPE}_{\mathrm{He}}=0.67 \%$ for the realistic case. This might be attributed to the minor but acceptable offset in figure 9 which is not present in figure 3 of Volz et al (2021). A previously developed artefact correction method for pCT (Dickmann et al 2021c) might correct this offset but was not applied to our data since the extension of the comparison of pCT and HeCT to artefact correction methods was beyond the scope of this study.

\section{Conclusions}

In this study, we presented a comparison of pCT and HeCT using the Monte Carlo simulation of the phase-II pCT scanner using both ideal and realistic scoring. As expected, for both ideal and realistic cases, helium had a higher resolution. At the center of the phantom, the ideal factor 2 resolution advantage of helium was slightly reduced to 1.8 in the realistic case due to limitations attributed to the tracking detectors. For the phase-II pCT scanner, the increased resolution of helium came at the cost of a factor 2.9 higher dose when matching noise. When equalizing the resolution of helium to that of protons by reconstruction kernel windowing, helium necessitated only $38 \%$ of the proton dose to produce equivalent noise. Finally, RSP accuracy was found to be comparable between the ions with MAPE of $0.59 \%$ for protons and $0.67 \%$ for helium. Overall, our study indicates a slightly superior performance of helium ions in particle imaging in terms of dose, variance and spatial resolution. HeCT might therefore help reduce the dose exposure of patients with image noise that is equal or reduced compared to $\mathrm{pCT}$, good spatial resolution and acceptable RSP accuracy at the same time.

\section{Acknowledgments}

We gratefully acknowledge the support of the U.S. pCT collaboration throughout this project, in particular Robert Johnson and Mark Pankuch. This work was supported by the German Research Foundation (DFG) project \#388731804 'Fluence modulated proton computed tomography: a new approach for low-dose image guidance in particle therapy' and the DFG's Cluster of Excellence Munich-Centre for Advanced Photonics (MAP). Additional funding from the Zusatzfinanzierung 2019 für DFG-Sachbeihilfen from the Forschungsdekanat of the Faculty of Medicine of the LMU Munich is acknowledged.

\section{Declaration of interests}

No conflict of interests to declare.

\section{Ethical statement}

No ethical statement required.

\section{Appendix}

\section{$\Delta E-E$ filter parameters}

Table A. 1 shows the parameters for the $\Delta E-E$ filter used in this work.

Table A.1. Parameters of the $\Delta E-E$ filter for stopping stages $2-5$. The indices low/high indicate if the parameter belongs to the lower/higher boundary of the filter.

\begin{tabular}{lcccccc}
\hline Stage & $a_{\text {low }} / \frac{1}{\mathrm{MeV}}$ & $b_{\text {low }}$ & $c_{\text {low }} / \mathrm{MeV}$ & $a_{\text {high }} / \frac{1}{\mathrm{MeV}}$ & $b_{\text {high }}$ & $c_{\text {high }} / \mathrm{MeV}$ \\
\hline 2 & $8.52 \times 10^{-4}$ & -0.569 & 225 & $8.41 \times 10^{-4}$ & -0.565 & 247 \\
3 & $8.52 \times 10^{-4}$ & -0.569 & 225 & $8.41 \times 10^{-4}$ & -0.565 & 247 \\
4 & $8.52 \times 10^{-4}$ & -0.569 & 232 & $8.41 \times 10^{-4}$ & -0.565 & 254 \\
5 & $4.36 \times 10^{-4}$ & -0.409 & 235 & $4.46 \times 10^{-4}$ & -0.413 & 260 \\
\hline
\end{tabular}


Table A.2. MLP parameters for helium ions according to equation (29) in Schulte et al (2008).

\begin{tabular}{|c|c|c|c|c|c|}
\hline$a_{0} / \frac{c^{2}}{\mathrm{MeV}^{2}}$ & $a_{1} / \frac{c^{2}}{\mathrm{MeV}^{2} \mathrm{~cm}}$ & $a_{2} / \frac{c^{2}}{\mathrm{MeV}^{2} \mathrm{~cm}^{2}}$ & $a_{3} / \frac{c^{2}}{\mathrm{MeV}^{2} \mathrm{~cm}^{3}}$ & $a_{4} / \frac{c^{2}}{\mathrm{MeV}^{2} \mathrm{~cm}^{4}}$ & $a_{5} / \frac{c^{2}}{\mathrm{MeV}^{2} \mathrm{~cm}^{5}}$ \\
\hline $4.666 \times 10^{-7}$ & $2.817 \times 10^{-8}$ & $-3.535 \times 10^{-9}$ & $7.964 \times 10^{-10}$ & $-5.636 \times 10^{-11}$ & $1.635 \times 10^{-12}$ \\
\hline
\end{tabular}

\section{Helium MLP parameters}

The MLP parameters for Helium ions are found in table A.2.

\section{Variance reconstruction accounting for kernel windowing}

Assuming a detector array of $D$ elements with width $\Delta \xi$, for parallel beam filtered backprojection with a given reconstruction kernel $g$ which in our study is obtained by the inverse Fourier transform of equation (2)

$$
g(\xi)=\int d p G_{\text {Hann }}(p) e^{2 \pi i p \xi}
$$

and number of projections $N_{P}$ from angle 0 to $\pi$, Rädler et al (2018) (which was in turn adapted from Wunderlich and Noo 2008) gives us the the following equation for the variance $\operatorname{Var}[f(x, y)]$ of a $2 \mathrm{D}$ image $f(x, y)$ for a pixel centered at $x$ and $y$

$$
\begin{aligned}
\operatorname{Var}[f(x, y)]= & \left(\frac{\pi \Delta \xi}{N_{P}}\right)^{2} \sum_{n=1}^{N_{P}}\left\{(1-u)^{2} \cdot V\left(\theta_{n}, j \Delta \xi\right)\right. \\
& +2(1-u) u \cdot C\left(\theta_{n}, j \Delta \xi,(j+1) \Delta \xi\right) \\
& \left.+u^{2} \cdot V\left(\theta_{n},(j+1) \Delta \xi\right)\right\},
\end{aligned}
$$

where $j \Delta \xi$ and $(j+1) \Delta \xi$ are the positions of the detector elements neighbouring the detector position $\xi(x, y)$ from which the projection variance value $\sigma_{p}^{2}\left(\theta_{n}, \xi\right)$ needs to be interpolated for projection angle $\theta_{n}$. The interpolation weight $u$ corresponds to the distance from the center of the neighbouring detector element with the lower index to $\xi(x, y)$. The prefactor $\frac{\pi \Delta \xi}{N_{P}}$ comes from the definition of the filtered backprojection. $V$ and $C$ are variance and covariance terms which are defined as

$$
\begin{gathered}
V\left(\theta_{n}, j \Delta \xi\right)=\sum_{m=-D / 2}^{D / 2-1} g^{2}((j-m) \Delta \xi) \cdot \sigma_{p}^{2}\left(\theta_{n}, m \Delta \xi\right), \\
C\left(\theta_{n}, j \Delta \xi,(j+1) \Delta \xi\right)=\sum_{m=-D / 2}^{D / 2-1} g_{C}((j-m) \Delta \xi) \cdot \sigma_{p}^{2}\left(\theta_{n}, m \Delta \xi\right),
\end{gathered}
$$

with

$$
g_{C}(j \Delta \xi)=g(j \Delta \xi) \cdot g((j+1) \Delta \xi) .
$$

As in Rädler et al (2018), we can assume that the convolution kernels $g^{2}$ and $g_{C}$ both fall quickly to zero and that $\sigma_{p}^{2}\left(\theta_{n}, \xi\right)$ is locally constant, allowing the following factorization

$$
\begin{gathered}
V\left(\theta_{n}, j \Delta \xi\right) \approx \sigma_{p}^{2}\left(\theta_{n}, j \Delta \xi\right) \cdot \sum_{m=-D / 2}^{D / 2-1} g^{2}(m \Delta \xi)=\sigma_{p}^{2}\left(\theta_{n}, j \Delta \xi\right) \cdot S_{g^{2}}, \\
C\left(\theta_{n}, j \Delta \xi,(j+1) \Delta \xi\right) \approx \sigma_{p}^{2}\left(\theta_{n}, j \Delta \xi\right) \cdot \sum_{m=-D / 2}^{D / 2-1} g_{C}(m \Delta \xi)=\sigma_{p}^{2}\left(\theta_{n}, j \Delta \xi\right) \cdot S_{g_{C}}
\end{gathered}
$$

With the above we can thus rewrite equation (A.2) as

$$
\operatorname{Var}[f(x, y)] \approx\left(\frac{\pi \Delta \xi}{N_{P}}\right)^{2} \sum_{n=1}^{N_{P}} \underbrace{\sigma_{p}^{2}\left(\theta_{n}, j \Delta \xi\right) S_{g^{2}}}_{V\left(\theta_{n}, j \Delta \xi\right)} \underbrace{\left\{(1-u)^{2}+2(1-u) u \frac{S_{g_{C}}}{S_{g^{2}}}+u^{2}\right\}}_{f_{\text {interp }}(u)},
$$

where $f_{\text {interp }}(u)$ is an interpolation factor depending only on $u$ and the sum of the filters $S_{g}^{2}$ and $S_{g_{C}}$. Note that we can use equation (A.6) to rewrite equation (A.8) as a convolution; we do this to exploit existing reconstruction software as explained below. We can further make a similar approximation as in Rädler et al (2018) and factor out the mean value of $f_{\text {interp }}(u)$

$$
\bar{f}_{\text {interp }}=\int_{0}^{1} d u f_{\text {interp }}(u)=\frac{2}{3}+\frac{1}{3} \frac{S_{g_{C}}}{S_{g^{2}}},
$$

which ignores the high frequency pattern caused by interpolation in $\operatorname{Var}[f(x, y)]$, but maintains its impact on the overall variance level. The final variance reconstruction used in this work is thus 


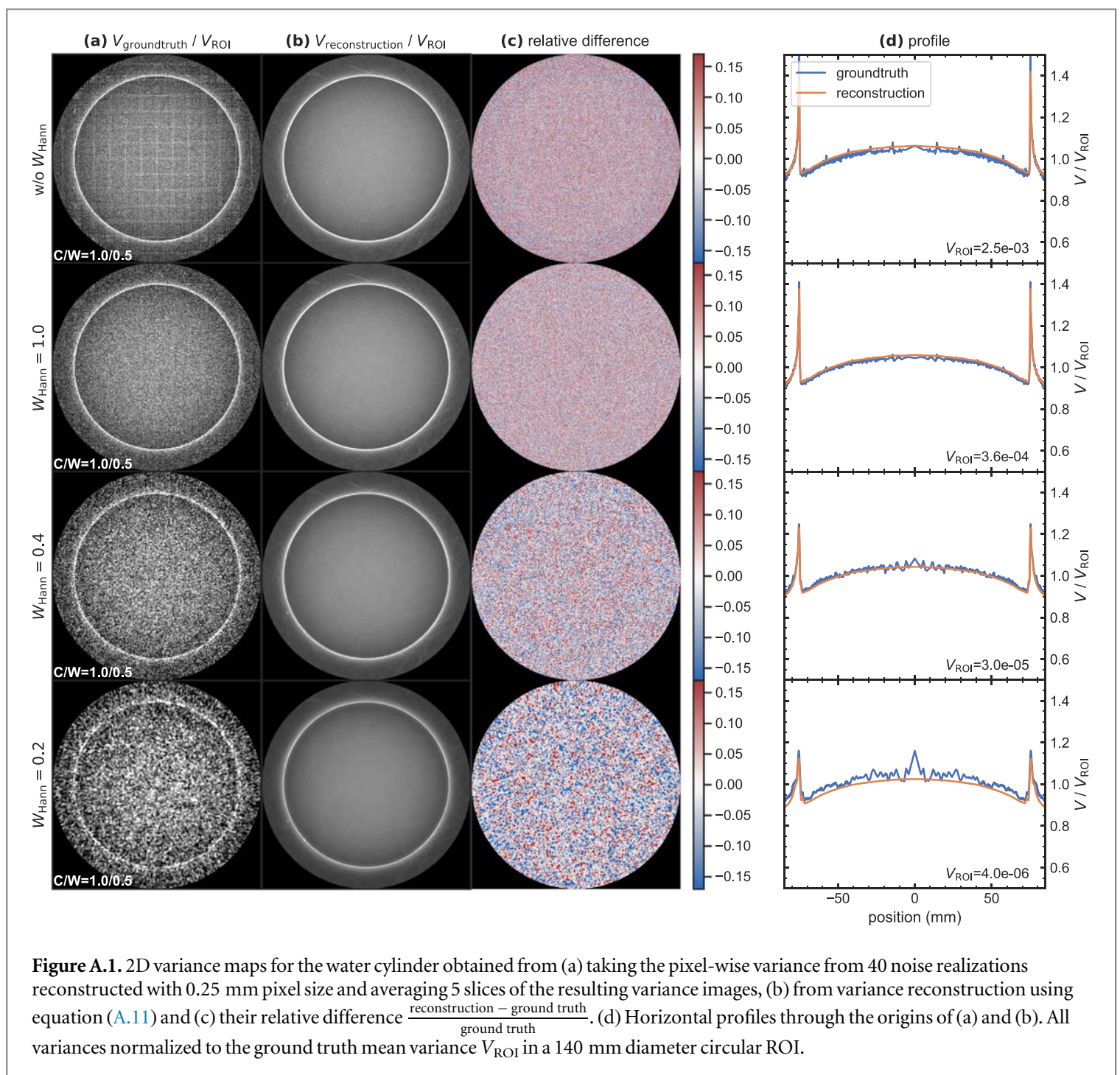

$$
\begin{gathered}
\operatorname{Var}[f(x, y)] \approx \bar{f}_{\text {interp }}\left(\frac{\pi \Delta \xi}{N_{P}}\right)^{2} \sum_{n=1}^{N_{P}} V\left(\theta_{n}, j \Delta \xi\right), \\
\operatorname{Var}[f(x, y)] \approx \bar{f}_{\text {interp }}\left(\frac{\pi \Delta \xi}{N_{P}}\right)^{2} \sum_{n=1}^{N_{P}} \sum_{m=-D / 2}^{D / 2-1} g^{2}((j-m) \Delta \xi) \cdot \sigma_{p}^{2}\left(\theta_{n}, m \Delta \xi\right),
\end{gathered}
$$

which allows realization in RTK by using the existing filtered backprojection implementation and replacing projection values by $\sigma_{p}^{2}\left(\theta_{n}, \xi\right)$, taking the square of the prefactor $\frac{\pi \Delta \xi}{N_{p}}$ and reconstruction kernel $g$, and computing the ratio $\frac{S_{g_{C}}}{S_{g^{2}}}$, which depends on $W_{\text {Hann }}$ to obtain $\bar{f}_{\text {interp }}$.

\section{Validation of variance reconstruction for Hann windowing}

To validate the approximations used to obtain equation (A.11), ground truth variance images were obtained by using 40 independent noise realizations with $0.25 \mathrm{~mm}$ pixel size for $\mathrm{pCT}$ and HeCT ideal simulated scans of the water phantom, similarly to Dickmann et al (2019). The results of this evaluation are presented for protons in figure A.1, and table A.3 shows the ratio of the variance reconstruction over the 40 independent realizations ground truth for different $W_{\text {Hann }}$ values. 
Table A.3. Variance ratios

of reconstruction to ground truth within a central ROI of $140 \mathrm{~mm}$ diameter of the water phantom for different Hann filter windows $W_{\text {Hann }}$.

\begin{tabular}{lc}
\hline$W_{\text {Hann }}$ & variance ratio \\
\hline $\mathrm{w} / \mathrm{o}$ & 1.01 \\
1.0 & 1.01 \\
0.4 & 0.99 \\
0.2 & 0.98 \\
\hline
\end{tabular}

\section{ORCID iDs}

S Götz (10) https://orcid.org/0000-0002-7518-7055

J Dickmann (ib https://orcid.org/0000-0001-8410-3995

N Krah (ii) https://orcid.org/0000-0002-1376-6633

F Khellaf (iD https://orcid.org/0000-0002-4266-0539

RW Schulte (i) https://orcid.org/0000-0002-7892-2756

K Parodi (i) https://orcid.org/0000-0001-7779-6690

G Landry (i) https://orcid.org/0000-0003-1707-4068

\section{References}

Agostinelli S et al 2003 Geant4-a simulation toolkit Nucl. Instrum. Methods Phys. Res. A 506 250-303

Bashkirov V A, Schulte R W, Hurley R F, Johnson R P, Sadrozinski H F-W, Zatserklyaniy A, Plautz T and Giacometti V 2016 Novel scintillation detector design and performance for proton radiography and computed tomography Med. Phys. 43 664-74

Berthold J et al 2021 First-in-human validation of CT-based proton range prediction using prompt gamma imaging in prostate cancer treatments Int. J. Radiat. Oncol., Biol., Phys. 111 1033-43

Birks J B 1951 Scintillations from organic crystals: specific fluorescence and relative response to different radiations Proc. Phys. Soc. London A $64874-7$

Bär E, Lalonde A, Zhang R, Jee K-W, Yang K, Sharp G, Liu B, Royle G, Bouchard H and Lu H-M 2018 Experimental validation of two dualenergy CT methods for proton therapy using heterogeneous tissue samples Med. Phys. 45 48-59

Bär E, Volz L, Collins-Fekete C-A, Brons S, Runz A, Schulte R W and Seco J 2021 Experimental comparison of photon versus particle computed tomography to predict tissue relative stopping powers Med. Phys. 49 474-87

Civinini C, Scaringella M, Brianzi M, Intravaia M, Randazzo N, Sipala V, Rovituso M, Tommasino F, Schwarz M and Bruzzi M 2020 Relative stopping power measurements and prosthesis artifacts reduction in proton CT Phys. Med. Biol. 65225012

Collins-Fekete C-A, Dikaios N, Bär E and Evans P M 2021 Statistical limitations in ion imaging Phys. Med. Biol. 66105009

Cormack A M 1964 Representation of a function by Its line integrals, with some radiological applications II J. Appl. Phys. 35 2908-13

Coutrakon G, Bashkirov V, Hurley F, Johnson R, Rykalin V, Sadrozinski H and Schulte R 2013 Design and construction of the I proton CT scanner AIP Conf. Proc. 1525 327-31

Dedes G et al 2017 Application of fluence field modulation to proton computed tomography for proton therapy imaging Phys. Med. Biol. 62 $6026-43$

Dedes G et al 2020 The role of Monte Carlo simulation in understanding the performance of proton computed tomography Z. Med. Phys. (https://doi.org/10.1016/j.zemedi.2020.06.006)

Dedes G et al 2019 Experimental comparison of proton CT and dual energy x-ray CT for relative stopping power estimation in proton therapy Phys. Med. Biol. 64165002

Dickmann J, Kamp F, Hillbrand M, Corradini S, Belka C, Schulte R W, Parodi K, Dedes G and Landry G 2021b Fluence-modulated proton CT optimized with patient-specific dose and variance objectives for proton dose calculation Phys. Med. Biol. 66064001

Dickmann J, Rit S, Pankuch M, Johnson R P, Schulte R W, Parodi K, Dedes G and Landry G 2020a An optimization algorithm for dose reduction with fluence-modulated proton CT Med. Phys. 47 1895-906

Dickmann J, Sarosiek C, Götz S, Pankuch M, Coutrakon G, Johnson R P, Schulte R W, Parodi K, Landry G and Dedes G 2021c An empirical artifact correction for proton computed tomography Phys. Medica: Eur. J. Med. Phys. 86 57-65

Dickmann J et al 2021a Proof of concept image artifact reduction by energy-modulated proton computed tomography (EMpCT) Phys. Medica: Eur. J. Med. Phys. 81 237-44

Dickmann J et al 2020b Experimental realization of dynamic fluence field optimization for proton computed tomography Phys. Med. Biol. 65 195001

Dickmann J et al 2019 Prediction of image noise contributions in proton computed tomography and comparison to measurements Phys. Med. Biol. 64145016

Durante M and Paganetti H 2016 Nuclear physics in particle therapy: a review Rep. Prog. Phys. 79096702

Esposito M et al 2018 PRaVDA: the first solid-state system for proton computed tomography Phys. Med. 55 149-54

Giacometti V et al 2017b Software platform for simulation of a prototype proton CT scanner Med. Phys. 44 1002-16

Giacometti V, Guatelli S, Bazalova-Carter M, Rosenfeld A and Schulte R 2017a Development of a high resolution voxelised head phantom for medical physics applications Phys. Med. 33 182-8 
Gottschalk B, Koehler A, Schneider R, Sisterson J and Wagner M 1993 Multiple Coulomb scattering of 160 MeV protons Nucl. Instrum. Methods Phys. Res. B 74 467-90

Hansen D, Bassler N, Sørensen T and Seco J 2014 The image quality of ion computed tomography at clinical imaging dose levels Med. Phys. 41111908

Hanson K M 1979 Proton computed tomography IEEE Trans. Nucl. Sci. 26 1635-40

Hünemohr N, Krauss B, Tremmel C, Ackermann B, Jäkel O and Greilich S 2013 Experimental verification of ion stopping power prediction from dual energy CT data in tissue surrogates Phys. Med. Biol. 59 83-96

Hudobivnik $\mathrm{N}$ et al 2016 Comparison of proton therapy treatment planning for head tumors with a pencil beam algorithm on dual and single energy CT images Med. Phys. 43 495-504

Johnson R P et al 2016 A fast experimental scanner for proton CT: technical performance and first experience with phantom scans IEEE Trans. Nucl. Sci. 63 52-60

Khellaf F, Krah N, Letang J-M, Collins-Fekete C-A and Rit S 2020 A comparison of direct reconstruction algorithms in proton computed tomography Phys. Med. Biol. 65105010

Krah N, Khellaf F, Létang J M, Rit S and Rinaldi I 2018 A comprehensive theoretical comparison of proton imaging set-ups in terms of spatial resolution Phys. Med. Biol. 63135013

Meyer S, Gianoli C, Magallanes L, Kopp B, Tessonnier T, Landry G, Dedes G, Voss B and Parodi K 2017 Comparative Monte Carlo study on the performance of integration- and list-mode detector configurations for carbon ion computed tomography Phys. Med. Biol. 62 1096-112

Meyer S, Kamp F, Tessonnier T, Mairani A, Belka C, Carlson D J, Gianoli C and Parodi K 2019 Dosimetric accuracy and radiobiological implications of ion computed tomography for proton therapy treatment planning Phys. Med. Biol. 64125008

Meyer S, Pinto M, Parodi K and Gianoli C 2021 The impact of path estimates in iterative ion CT reconstructions for clinical-like cases Phys. Med. Biol. 66095007

Niepel K B et al 2021 Animal tissue-based quantitative comparison of dual-energy CT to SPR conversion methods using high-resolution gel dosimetry Phys. Med. Biol. 66075009

Paganetti H 2012 Range uncertainties in proton therapy and the role of Monte Carlo simulations Phys. Med. Biol. 57 R99-117

Park H, Paganetti H, Schuemann J, Jia X and Min C H 2021 Monte Carlo methods for device simulations in radiation therapy Phys. Med. Biol. 66 18TR01

Pettersen $\mathrm{H}$ et al 2017 Proton tracking in a high-granularity digital tracking calorimeter for proton CT purposes, Nucl. Instrum. Methods Phys. Res. A $86051-61$

Piersimoni P, Faddegon B A, Méndez J R, Schulte R W, Volz L and Seco J 2018 Helium CT: Monte Carlo simulation results for an ideal source and detector with comparison to proton CT Med. Phys. 45 3264-74

Piersimoni P, Ramos-Méndez J, Geoghegan T, Bashkirov V A, Schulte R W and Faddegon B A 2017 The effect of beam purity and scanner complexity on proton CT accuracy Med. Phys. 44 284-98

Rädler M, Landry G, Rit S, Schulte R W, Parodi K and Dedes G 2018 Two-dimensional noise reconstruction in proton computed tomography using distance-driven filtered back-projection of simulated projections Phys. Med. Biol. 63215009

Richard S, Husarik D B, Yadava G, Murphy S N and Samei E 2012 Towards task-based assessment of CT performance: System and object MTF across different reconstruction algorithms Med. Phys. 39 4115-22

Rinaldi I, Brons S, Gordon J, Panse R, Voss B, Jäkel O and Parodi K 2013 Experimental characterization of a prototype detector system for carbon ion radiography and tomography Phys. Med. Biol. 58 413-27

Rit S, Dedes G, Freud N, Sarrut D and Létang J M 2013 Filtered backprojection proton CT reconstruction along most likely paths Med. Phys. 40031103

Sadrozinski H-W et al 2016 Operation of the preclinical head scanner for proton CT Nucl. Instrum. Methods Phys. Res. A 831 394-9

Schulte R W, Bashkirov V, Klock M C, Li T, Wroe A J, Evseev I, Williams D C and Satogata T 2005 Density resolution of proton computed tomography Med. Phys. 32 1035-46

Schulte R W, Penfold S N, Tafas J T and Schubert K E 2008 A maximum likelihood proton path formalism for application in proton computed tomography Med. Phys. 35 4849-56

Taasti V T, Michalak G J, Hansen D C, Deisher A J, Kruse J J, Krauss B, Muren L P, Petersen J B B and McCollough C H 2017 Validation of proton stopping power ratio estimation based on dual energy CT using fresh tissue samples Phys. Med. Biol. 63015012

Tilley S, Siewerdsen J H and Stayman J W 2015 Model-based iterative reconstruction for flat-panel cone-beam CT with focal spot blur, detector blur, and correlated noise Phys. Med. Biol. 61 296-319

Volz L 2021 Particle imaging for daily in-room image guidance in particle therapy PhD Thesis Universität Heidelberg (http://ub.uniheidelberg.de/archiv/29273)

Volz L, Collins-Fekete C-A, Bär E, Brons S, Graeff C, Johnson R P, Runz A, Sarosiek C, Schulte R W and Seco J 2021 The accuracy of helium ion CT based particle therapy range prediction: an experimental study comparing different particle and x-ray CT modalities Phys. Med. Biol. 66235010

Volz L, Piersimoni P, Bashkirov V A, Brons S, Collins-Fekete C-A, Johnson R P, Schulte R W and Seco J 2018 The impact of secondary fragments on the image quality of helium ion imaging Phys. Med. Biol. 63195016

Wohlfahrt P, Möhler C, Stützer K, Greilich S and Richter C 2017 Dual-energy CT based proton range prediction in head and pelvic tumor patients Radiother. Oncol. 125 526-33

Wunderlich A and Noo F 2008 Image covariance and lesion detectability in direct fan-beam x-ray computed tomography Phys. Med. Biol. 53 2471-93

Yang M, Virshup G, Clayton J, Zhu X R, Mohan R and Dong L 2010 Theoretical variance analysis of single- and dual-energy computed tomography methods for calculating proton stopping power ratios of biological tissues Phys. Med. Biol. 55 1343-62

Yang M, Zhu X R, Park P C, Titt U, Mohan R, Virshup G, Clayton J E and Dong L 2012 Comprehensive analysis of proton range uncertainties related to patient stopping-power-ratio estimation using the stoichiometric calibration Phys. Med. Biol. 57 4095-115 